\title{
Time integrative passive sampling of very hydrophilic chemicals in wastewater influent
}

Rory Verhagen ${ }^{1}$, Sarit L. Kaserzon ${ }^{1}$, Benjamin J. Tscharke ${ }^{1}$, Pradeep Shukla ${ }^{1}$,Joseph Clokey ${ }^{1}$, Branislav Vrana ${ }^{2}$, Kevin $\vee$ Thomas $^{1}$, and Jochen F Mueller ${ }^{1}$

${ }^{1}$ Queensland Alliance for Environmental Health Sciences, The University of Queensland, 20 Cornwall Street Woolloongabba, Queensland 4102, Australia

2 Masaryk University, Faculty of Science, Research Centre for Toxic Compounds in the Environment (RECETOX), Kamenice 753/5, 625 00, Brno, Czech Republic

\section{Corresponding Author: Rory Verhagen}
Ph: +61404477716
E: rory.verhagen@uq.edu.au

Supplementary information:

SI1: Materials and methods (page 2 and 3 )

SI2: Specifics G-TIPS passive sampler (page 4)

SI3: Graphs for the four different sampling duration sections $R \mathrm{~s}_{0-15}, R \mathrm{~s}_{7-21}, R \mathrm{~s}_{17-31}$ and $R \mathrm{~s}_{0-31}$ (page 5)

SI4: Concentration of analytes in wastewater derived from $24 \mathrm{~h}$ composite samples (page 6)

SI5: Explanation of theory used to predict sampling rates with the chemical diffusivity though water (page 7 and 8).

SI6: Calculated partitioning coefficients (page 9) 


\section{SI1 Materials and methods}

Materials. Methanol and acetone were purchased from Merck (Germany) and were of chromatography quality. Water was purified through a Milli-Q system (Millipore, $0.22 \mu \mathrm{m}$ filtered, $18.2 \mathrm{~m} \Omega \mathrm{cm}^{-1}$ ). Regenerated cellulose (RC) filters ( $0.2 \mathrm{~mm}$ pore size) were purchased from Agilent Technologies and used to filter the samples. Acesulfame, ethyl sulphate, methamphetamine, benzoylecgonine, acesulfame D4, ethyl sulphate D5, methamphetamine D9 and benzoylecgonine D3 were purchased from Novachem (Heidelberg West, Australia). A stock solution of $1000 \mu \mathrm{g} \mathrm{mL}^{-1}$ (native) and $100 \mu \mathrm{g} \mathrm{m}^{-1}$ (isotopically labelled) was prepared and stored at $-20^{\circ} \mathrm{C}$.

Deployment. The sampler was deployed over 31 days in the influent of a municipal wastewater treatment plant in Queensland, Australia. Location of sampling was after influent screening and grit removal processes within the WWTP. Analytical triplicates $1.5 \mathrm{~mL}$ were taken from the sampling port with a syringe each day for the first 7 days of deployment, followed by days: $11,15,17,21,24,28$ and 31 . The exact volume extracted from G-TIPS was replaced with clean milliQ-water via the sampling port. Concurrent to the deployment of the passive sampler, on each day, a refrigerated auto sampler collected a $24 \mathrm{~h}$ composite sample $(250 \mathrm{~mL})$ in time-proportional mode with 15 min intervals for the entire 31-day period.

Analysis. Aliquots of the samples collected from G-TIPS and the $24 \mathrm{~h}$ composite samples (1 $\mathrm{mL}$ ) were filtered using an RC $0.2 \mu \mathrm{m}$ Agilent filter on the day of collection (within an hour of sampling) and spiked with isotopically labelled standards (10 $\mu \mathrm{L}$ of a $1 \mathrm{ppm}$ stock solution) prior to direct injection analysis on LC-MS/MS. All samples were stored at $-20^{\circ} \mathrm{C}$ until analysis. Samples were analysed using Liquid Chromatography (LC) (Shimadzu, Nexera HPLC system, Kyoto, Japan) coupled with tandem electrospray ionization (ESI) mass spectrometry (MS/MS) (SCIEX API 5500 QTRAP ${ }^{\circledR}$ Mass Spectrometers, Ontario, Canada). To quantify the target analytes the isotopic ratio between native and labelled was used. An eight-point calibration (0.1-100 $\left.\mathrm{ng} \mathrm{mL}^{-1}\right)$ was used to calculate the concentrations in the samples. Data was processed with MultiQuant ${ }^{\mathrm{TM}}$ and Analyst Software (Sciex).

Quality assurance and quality control (QA/QC). Samples were collected from the sampler prior to deployment (time 0), and were considered as a field blanks. Two additional blanks were taken from a sample bottle brought into the field during the sampling period. None of the analytes of interest were detectable in the time 0/field blank samples. Results from the 
analysis of triplicate G-TIPS samples showed low percent coefficient of variation (\%CV) within the sampler $<16 \%$ (with the exception of day one for EtS at 35\%).

Analytical method detection limits (LODs) were calculated as three times the standard deviation of the 0.1 or $0.5 \mathrm{ng} \mathrm{mL}^{-1}$ calibration standard from $5 x$ injections. Limits of quantification (LOQs) were calculated as three times the LOD. LODs ranged from 0.003 to 0.15 $\mathrm{ng} \mathrm{mL} \mathrm{m}^{-1}$ for benzoylecgonine and acesulfame (table 1).

Our laboratory uses well-established quantitation methods for drugs, and routinely participates in passive sampling inter-laboratory studies and the EU wastewater based drug monitoring inter-laboratory comparison study SCORE ${ }^{1,2}$. 


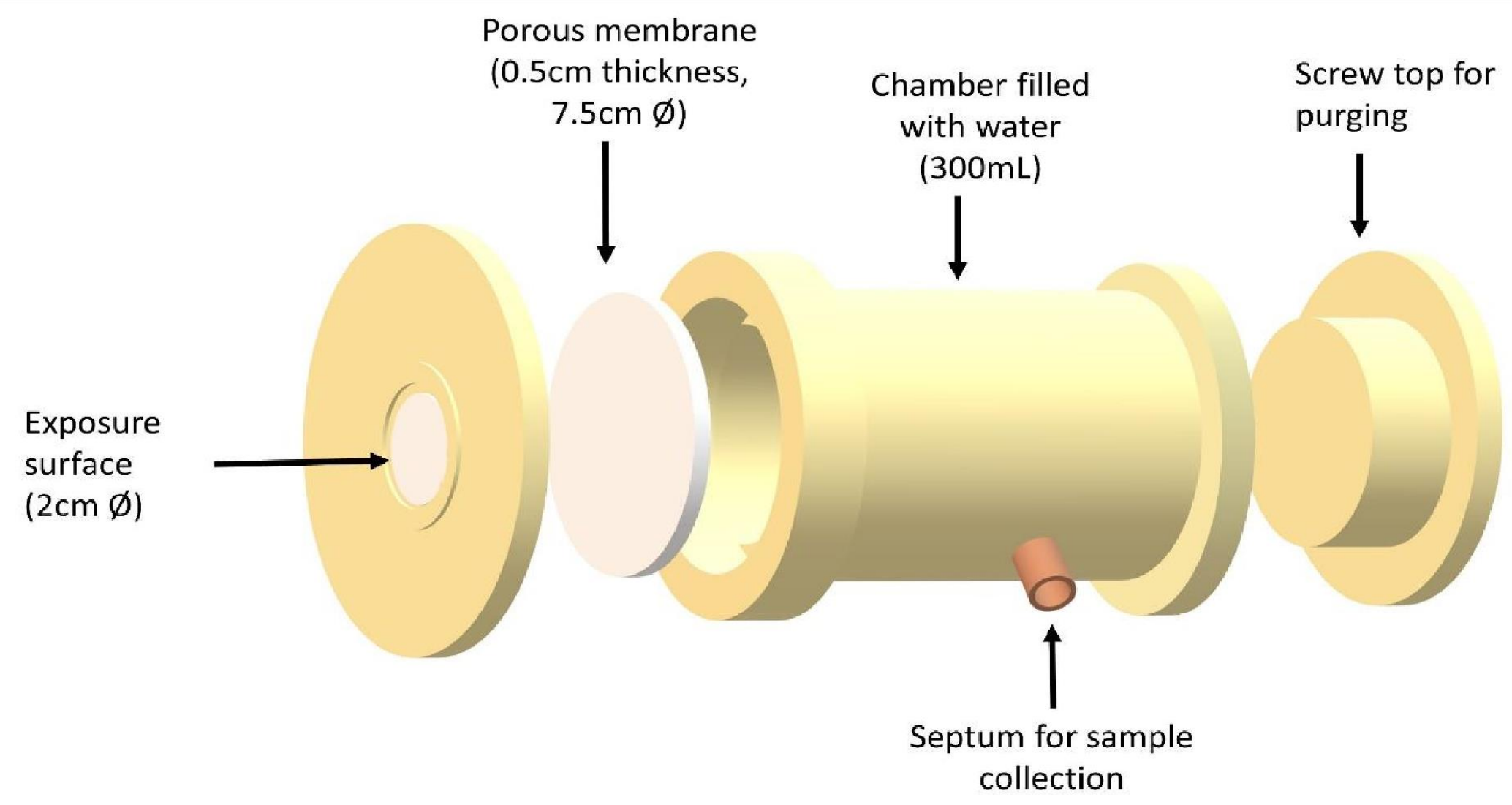


SI3: Calculated sampling rates (Eq3) for the four different sampling duration sections ( $R \mathrm{~s}_{0-15}, R \mathrm{~s}_{7-21}, R \mathrm{~s}_{17-31}$ and $R \mathrm{~s}_{0-31}$ On the left $\mathrm{y}$-axis the product of $C \mathrm{w} / \mathrm{Cs}_{\mathrm{s}}$ (circles), on the right $\mathrm{y}$-axis are the concentrations of the raw wastewater $\mathrm{ng} \mathrm{mL}^{-1}$ (squares) determined from the composite $24 \mathrm{~h}$ auto samplers and on the $x$-axis the time in days.
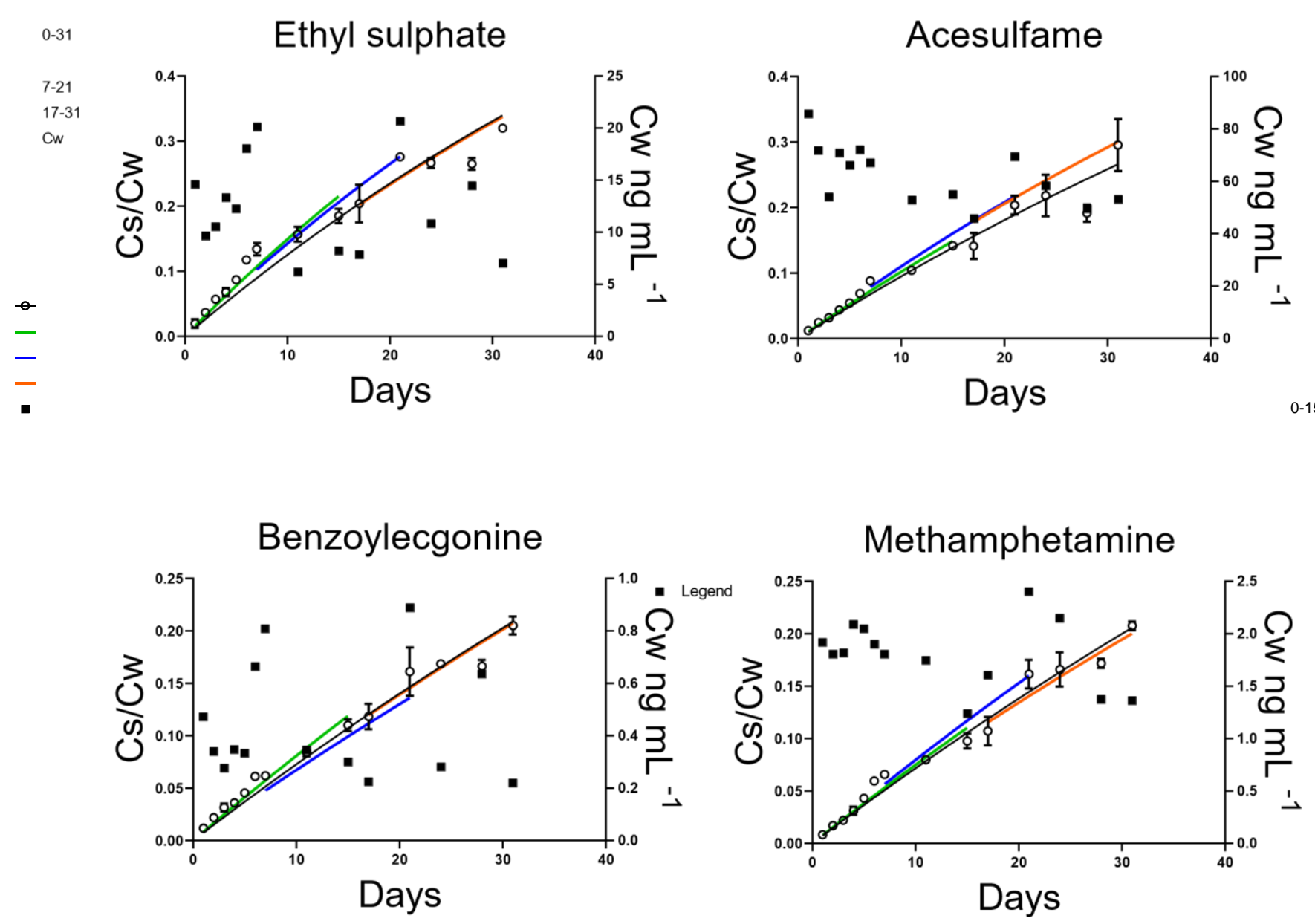
SI4: concentration of analytes in wastewater derived from $24 \mathrm{~h}$ composite samples

\begin{tabular}{rrrrr} 
Days & $\mathrm{EtS}\left(\mathrm{ng} \mathrm{mL}^{-1}\right)$ & $\mathrm{ACS}\left(\mathrm{ng} \mathrm{mL}^{-1}\right)$ & $\mathrm{BZE}\left(\mathrm{ng} \mathrm{mL}^{-1}\right)$ & Meth $\left(\mathrm{ng} \mathrm{mL}^{-1}\right)$ \\
\hline 1 & 14.6 & 85.6 & 0.5 & 1.9 \\
2 & 9.6 & 71.9 & 0.3 & 1.8 \\
3 & 10.5 & 54.0 & 0.3 & 1.8 \\
4 & 13.3 & 70.9 & 0.3 & 2.1 \\
5 & 12.3 & 66.1 & 0.3 & 2.0 \\
6 & 18.0 & 72.1 & 0.7 & 1.9 \\
7 & 20.1 & 67.0 & 0.8 & 1.8 \\
11 & 6.2 & 52.9 & 0.3 & 1.7 \\
15 & 8.2 & 55.0 & 0.3 & 1.2 \\
17 & 7.8 & 45.8 & 0.2 & 1.6 \\
21 & 20.6 & 69.5 & 0.9 & 2.4 \\
24 & 10.8 & 58.4 & 0.3 & 2.1 \\
28 & 14.5 & 49.6 & 0.6 & 1.4 \\
31 & 7.0 & 53.1 & 0.2 & 1.4
\end{tabular}


SI5: Explanation of theory used to predict sampling rates with the chemical diffusivity though water.

One of the reasons porous polyethylene is useful as a diffusive barrier is that water-filled pores in PE have similar properties to stagnant water. The molecules diffusing from the sampled aqueous phase across the porous polyethylene membrane to the receiving aqueous phase pass several barriers to mass transfer. Besides the membrane pores water boundary layers (WBL) can form on both sides of the membrane. The overall resistance to mass transfer can be expressed a sum of resistances of individual barriers in series:

$\frac{1}{k_{o}}=\frac{1}{k_{w, e x t}}+\frac{1}{k_{P E}}+\frac{1}{k_{w, i n t}}$

Where $k_{\mathrm{o}}$ is the overall mass transfer coefficients, $k_{\mathrm{w}, \text { ext }}$ and $k_{\mathrm{w}, \text { int }}$ are resistances of the WBL on external and internal side of the PE membrane, respectively. The mass transfer in PE controls the uptake of sampled compounds when the WBL resistances are negligible, i.e. when

$\frac{1}{k_{P E}} \gg \frac{1}{k_{w, e x t}}+\frac{1}{k_{w, i n t}}$

To assure this, water on both sides of PE membrane should be stirred to minimise the thickness of WBL or creation of undesired concentration gradients in liquid phases at both sides of PE membrane surface. We assume that the compounds pass the membrane via the water-filled pores and due to their very hydrophilic character they do not interact with the polymer matrix of PE. In such case, $k_{\mathrm{PE}}$ can be estimated as ${ }^{3}$ :

$k_{P E}=\frac{\phi D_{w}}{\theta \delta_{P E}}$

where $\phi$ is the porosity, $\theta$ the tortuosity of the diffusion pathways within the PE membrane and $\delta_{P E}$ is the PE membrane thickness.

$D_{w}$ values can be estimated, e.g. using the Wilke-Chang equation ${ }^{4}$ :

$D=7.4 \times 10^{-8} \frac{(x M)^{0.5} T}{\eta V^{0.6}}$

or the Hayduk-Laudie equation ${ }^{5}$ :

$D=\frac{13.26 \times 10^{-5}}{\eta^{1.4} V^{0.589}}$

where $x$ is the solvent association parameter ( 2.6 for water), $M\left(\mathrm{~g} \mathrm{~mol}^{-1}\right)$ is molar mass of water, $T(\mathrm{~K})$ is the thermodynamic temperature, $\eta(\mathrm{cP})$ is viscosity of water and $V\left(\mathrm{~cm}^{3} \mathrm{~mol}^{-1}\right)$ is molar volume of the diffusing compound at its normal boiling point.

Estimated $D_{w}$ at $23^{\circ} \mathrm{C}$. 


\begin{tabular}{lrrrr} 
Compound & Molar & $\begin{array}{l}\text { Molar } \\
\text { volume }\end{array}$ & $\begin{array}{l}\text { Wilke- } \\
\text { Chang } \\
\mathrm{D} \times 10^{6} \\
\mathrm{~cm}^{2} \mathrm{~s}^{-1}\end{array}$ & $\begin{array}{l}\text { Hayduk- } \\
\text { Laudie } \\
\mathrm{D} \times 10^{6} \\
\mathrm{~cm}^{2} \mathrm{~s}^{-1}\end{array}$ \\
\hline Acesulfame & 201.242 & 111.18 & 9.54 & 8.98 \\
Ethyl sulphate & 125.99 & 83.99 & 11.29 & 10.6 \\
Methamphetamine & 149.23 & 165.81 & 7.51 & 7.1 \\
Benzoylecgonine & 289.33 & 222.56 & 6.29 & 5.97
\end{tabular}

The porosity is know from the manufacture and is $35 \%$.

The theoretical tortuosity for a randomly oriented cylindrical pore network can be determined as follow ${ }^{6,7}$.

The diffusion flux along the pore aligned in direction " $\mathrm{R}$ " of the liquid flux is given as $F=-D \frac{d C}{d R}$. Assuming that the pore axis is at an angle $\varnothing$ from the " $z$ " axis, or the direction of net liquid flow. Hence $\mathrm{dz}=\mathrm{dR} \cos \varnothing$.

Additionally, the component of flux in the $z$ direction is given as $F_{z}=-D \cos \emptyset \frac{d C}{d R}$, where $\mathrm{Fz}$ is the $\mathrm{z}$ component of the flux.

Combining 6 and 7 , we get $F_{z}=-D \cos ^{2} \emptyset \frac{d C}{d R^{\prime}}$, thus tortuosity is represented as $1 / \cos ^{2} \emptyset$

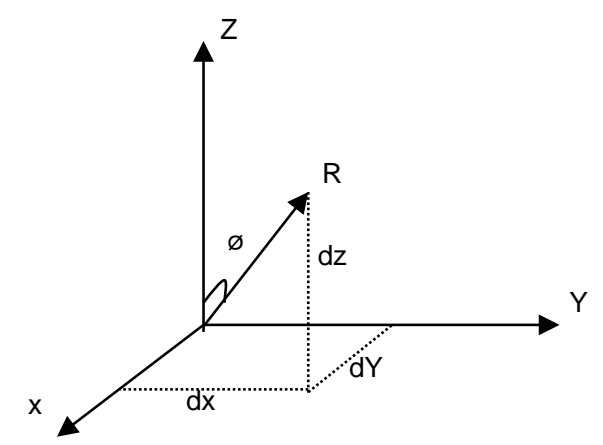
For a randomly oriented pore and for a very large sample, the "average value" of distance $\overline{d R}$ can be equated to $\overline{d R \cos \emptyset}=\overline{d z}=\overline{d x}=\overline{d y}$ Also since $d r^{2}=d z^{2}+d x^{2}+d y^{2}$. Combining we get the averaged values, $\mathrm{dr}^{2}=3 \overline{\cos ^{2} \varnothing} \mathrm{dr} r^{2}$. Or $\overline{\cos ^{2} \varnothing}=\frac{1}{3}$. Thus tortuosity for a randomly oriented pore equals 3.0.

The sampling rate can be estimated as $R_{\mathrm{S}}=k_{\mathrm{PE}} A$ :

$R_{S}=k_{P E} A=\frac{\phi D_{w} A}{\theta \delta_{P E}}$

where $A$ is the surface area of sampler in contact with sampled water $\left(30.19 \mathrm{~cm}^{2}\right), \delta_{\mathrm{PE}}=0.5 \mathrm{~cm}$, $\phi=0.35$ and $\theta=3$ the estimated Rs values are:

\begin{tabular}{lcc} 
Compound & $\begin{array}{l}\text { Rs } \\
\text { (mL/day) } \\
\text { (Wilke } \\
\text { Chang) }\end{array}$ & $\begin{array}{l}\text { Rs } \\
\text { (mL/day) } \\
\text { (Hayduk- } \\
\text { Laudie) }\end{array}$ \\
\hline Acesulfame & 5.81 & 5.47 \\
Ethyl sulphate & 6.87 & 6.45 \\
Methamphetamine & 4.57 & 4.32 \\
Benzoylecgonine & 3.83 & 3.63
\end{tabular}


SI6: Calculated partition coefficients from unpublished work showing no interaction with the PE membrane as partition coefficients are close to 1 or no chemicals were detected in the PE membrane (a).

\begin{tabular}{lc} 
Compound & $\begin{array}{c}K_{\mathrm{mw}} \\
\left(\mathrm{mL} \mathrm{g}^{-1}\right)\end{array}$ \\
\hline \hline Acesulfame & $\mathrm{a}$ \\
Codeine & $1.19 \pm 0.12$ \\
lbuprofen & $1.06 \pm 0.11$ \\
Naproxen & $0.68 \pm 0.02$ \\
Paracetamol & $\mathrm{a}$
\end{tabular}

1. Vrana, B.; Smedes, F.; Prokeš, R.; Loos, R.; Mazzella, N.; Miege, C.; Budzinski, H.;

Vermeirssen, E.; Ocelka, T.; Gravell, A., An interlaboratory study on passive sampling of emerging water pollutants. TrAC 2016, 76, 153-165.

2. Hageman, K. J.; Aebig, C. H.; Luong, K. H.; Kaserzon, S. L.; Wong, C. S.; Reeks, T.;

Greenwood, M.; Macaulay, S.; Matthaei, C. D., Current-use pesticides in New Zealand streams:

Comparing results from grab samples and three types of passive samplers. Environmental Pollution 2019, 254, 112973.

3. Booij, K.; Vrana, B.; Huckins, J. N., Theory, modelling and calibration of passive samplers used in water monitoring. Compr. Anal. Chem. 2007, 48, 141-169.

4. Chang, P.; Wilke, C., Some Measurements of Diffusion in Liquids. The Journal of Physical Chemistry 1955, 59 (7), 592-596.

5. Hayduk, W.; Laudie, H., Prediction of diffusion coefficients for nonelectrolytes in dilute aqueous solutions. AlChE Journal 1974, 20 (3), 611-615.

6. Johnson, M. F.; Stewart, W. E., Pore structure and gaseous diffusion in solid catalysts. J. Catal. 1965, 4 (2), 248-252.

7. Bhatia, S. K.; Perlmutter, D., A random pore model for fluid-solid reactions: I. Isothermal, kinetic control. AlChE Journal 1980, 26 (3), 379-386. 
